Guido LUPIERI

Giorgio CONTENTO

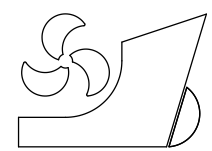

http://dx.doi.org/10.21278/brod67405

ISSN 0007-215X

eISSN 1845-5859

\title{
A NUMERICAL STUDY ON THE VISCOUS EFFECTS OF WAVES TRAVELLING PAST A WEAKLY SUBMERGED CYLINDER
}

UDC 629.5(05):629.5.015.2:629.5.015.024

Original scientific paper

\section{Summary}

The interaction of waves with a weakly submerged horizontal circular cylinder is studied numerically, solving 2-D Navier-Stokes equations with reference to former experimental sessions in a wave flume. A selection of aspects of the viscous wave-body interaction is analyzed, in particular the free surface elevation perturbed by the obstacle, the pressure at the body surface, the inertia coefficient of the Morison equation and finally the generation, persistence and merging of vorticity structures detached from the cylinder.

Key words: wavy flow; submerged circular cylinder; wave loads; merging eddies;

\section{Introduction}

The prediction of wave loads on fixed or floating objects is of interest in ship and off-shore engineering since related to the hydrodynamic design of vessels/structures customized according to the life-time expected sea states. In this panorama, the study of fluid-body interaction in case of a 2D orbital or wavy flows around a fully submerged cylinder of circular or squared shape stands as a fundamental case. Among the authors that contributed to these studies, the following are key references: Ogilvie [1], Longuet-Higgins [2], Riley [3], Riley [4], Vada [5], Stansby and Smith [6], Wu and Eatock Taylor [7], Stansby [8], Otsuka and Ikeda [9], Chaplin [10], Chaplin [11], Chaplin [12], Chaplin [13], Grue [14], Oshkai and Rockwell [15], Contento and Codiglia [16]. These studies could by subdivided into two main sub-subjects: diffraction and viscous effects respectively. In particular Ogilvie [1], Vada [5] and $\mathrm{Wu}$ and Eatock Taylor [7] have derived the expression for the second-order diffraction force by a perturbation expansion in the frame of the inviscid flow approximation. From these analyses the second order effects have been directly related to the submergence of the cylinder. In the viscous scenario, Longuet-Higgins [2], Riley [3] and Riley [4] have shown that a thorough modification of the external inviscid flow derives from the non-uniform oscillatory nature of the boundary layer around the cylinder that in turn induces a steady streaming in the proximity of the cylinder surface. Chaplin $[10,11]$ has conducted experimental tests measuring both the forces acting on the cylinder and the fluid velocity field in a region close to the cylinder surface. His results demonstrate the strongly nonlinear behaviour of the loads for Keulegan-Carpenter [17] numbers $K C$ less than 3. According to the measurements and to the theoretical results of Riley $[3,4]$, Chaplin $[10,11]$ has shown also that, provided the flow is not separated, the inertia 
term in the best-fit Morison equation [18] follows a linear plus a cubic function in $K C$ up to the separation limit, the coefficient of the cubic term being negative. Therefore the inertia coefficient $C_{m}$ behaves as a quadratic function of $K C$. This has been explained as the effect of the interaction between the steady streaming with the incident orbital flow (Magnus effect).

Otsuka and Ikeda [9] have presented some visualizations of the flow giving experimental evidence to the separation that occurs around the cylinder when the orbital uniform flow is started from rest. From these visualizations, they have derived a vortex shedding model that gives a simple quadratic law in $K C$ for the inertia coefficient $C_{m}$. Their theoretical results are in close agreement with those derived experimentally by Chaplin [11].

Oshkai and Rockwell [15] have conducted experimental tests with PIV and have analyzed the interaction between a free surface wavy flow and a submerged cylinder in terms of correlation between patterns of vorticity and loads, including the effect of submergence. Their tests were conducted with $K C$ around 7 . They have shown that the presence of the free surface can exert a remarkable influence on the initial formation, the strength, and the subsequent motion of concentration of vorticity.

Contento and Codiglia [16] have studied the interaction of regular waves past a submerged horizontal circular cylinder with different values of the submergence of the cylinder axis, respectively 2, 3 and 4 radii. The $K C$ range investigated in the experiments is between 0.1 and 2.5 , in laminar flow regime with a nominal Reynolds number $R e$ of order of 10,000. The diffraction parameter $k a$, where $k$ is the wave number and $a$ is the cylinder radius, has been varied between 0.125 and 0.52 . Wave loads have been obtained by pressure integrals over a large number of pressure probes, assuming that for such blunt body and at these $K C$ and $R e$, the tangential stress is orders of magnitude lower than the normal stress. Their results are in full agreements with those obtained by direct measurement of the force by Chaplin [10,11] and those obtained by Grue [14], both in terms of nonlinearities of the forces and of the free surface behavior. Among the results, they have shown that second and third order Fourier components of the surface pressure and of the free surface elevation in a wide portion at the lee side of the cylinder are strongly dependent on the submergence of the cylinder, whereas first order terms are only weakly dependent on it, consistently with the $k a$ values used.

Chaplin [19] conducted a series of experiments aimed at the identification of the non-linear features of wave interaction in the presence of a weakly submerged cylinder for a wide range of the diffraction parameter $k a$ and varying the incident wave amplitude up to breaking, comparing the results to inviscid flow solutions from the literature. In diffraction regime, second order solutions were shown to give excellent results in terms of wave profile on the lee side of the cylinder, necessarily without breaking. Higher frequencies up to the fifth were also detected in the measured wave elevation profile. The mean wave elevation above the cylinder was also derived from the measurements and checked against theoretical prediction with good results up to the breaking condition. Finally transmitted waves at the first, second and third harmonic were studied carefully, specifically their dependence on the incident wave amplitude. It was found that the second and third harmonic behave as a power of 2 and 3 respectively plus a contribution in the 4 th and 5 th power of the incident wave amplitude.

In the present work, the study has been conducted by integrating the Navier-Stokes equations. The intent is to evidence viscous effects, accounted for explicitly in the diffusive term, and to highlight non-linear effects related to the convective term. Forces and pressure at the cylinder and the free surface are analyzed by using the experimental results previously obtained by one of the authors [16]. In particular, the focus is initially on the wave elevation and on the forces at the cylinder surface and related inertia coefficient. Finally, the computation looks at some physics of the phenomenon that could not be directly evinced in the experiments, in particular the evolution of vorticity patterns detached from the cylinder. 


\section{Problem formulation}

In Fig. 1 a schematic representation of the physical problem is given, with the relevant symbols. A Cartesian frame of reference $O(x, y)$ is used with $x$-axis placed at the still free surface and the $y$-axis is upwardly oriented. The origin $O$ is placed in correspondence of the longitudinal position of the cylinder axis. It is assumed that the incident waves travel in the positive $x$-axis direction. The angular coordinate $\theta$ on the cylinder surface is used too, starting from the vertical position and with positive values in clockwise sense.

Adopting a linear wave model, the nominal Keulegan-Carpenter number $K C$ and the Reynolds number $R e$ read as follows:

$$
\begin{aligned}
& K C=\frac{U T}{D}=\frac{\pi H}{D} \frac{\cosh (k(y+h))}{\sinh (k h)} \\
& R e=\frac{\rho U D}{\mu}=\frac{\rho \pi H D}{\mu T} \frac{\cosh (k(y+h))}{\sinh (k h)}
\end{aligned}
$$

Here $U$ is the maximum velocity of the incident undisturbed flow at the cylinder axis, $T$ is the dominant period of the incident flow, $H$ is the wave height, $\lambda$ is the incident wavelength, $k$ is the wavenumber, $D=2 a$ is the cylinder diameter ( $a$ is the radius), $y$ is the depth of the cylinder axis and $h=1.550 \pm 0.002 \mathrm{~m}$ is the water depth assumed constant.

\begin{tabular}{|l|l|l|l|l|l|l|l|l|l|l|}
\hline & Test \# & 1 & 4 & 7 & 11 & 15 & 19 & 22 & 26 & 30 \\
\hline & $K C$ & 0.10 & 0.25 & 0.40 & 0.60 & 0.80 & 1.18 & 1.54 & 2.02 & 2.50 \\
\hline & $k a$ & 0.521 & 0.421 & 0.353 & 0.291 & 0.247 & 0.214 & 0.179 & 0.148 & 0.126 \\
\hline & $R e$ & 7937 & 17836 & 26123 & 35462 & 43372 & 21721 & 25947 & 30803 & 34969 \\
\hline & $D(\mathrm{~m})$ & 0.315 & 0.315 & 0.315 & 0.315 & 0.315 & 0.160 & 0.160 & 0.160 & 0.160 \\
\hline & $\lambda(\mathrm{m})$ & 1.90 & 2.35 & 2.80 & 3.40 & 4.00 & 2.35 & 2.80 & 3.40 & 4.00 \\
\hline & $T(\mathrm{~s})$ & 1.103 & 1.2271 & 1.339 & 1.4759 & 1.601 & 1.2271 & 1.339 & 1.4759 & 1.601 \\
\hline$y / a=-2$ & $H(\mathrm{~m})$ & 0.028 & 0.058 & 0.080 & 0.106 & 0.127 & 0.092 & 0.111 & 0.137 & 0.159 \\
\hline$y / a=-3$ & $H(\mathrm{~m})$ & 0.047 & 0.088 & 0.114 & 0.140 & 0.160 & 0.114 & 0.133 & 0.158 & 0.180 \\
\hline$y / a=-4$ & $H(\mathrm{~m})$ & 0.080 & 0.134 & 0.161 & 0.185 & 0.200 & 0.141 & 0.159 & 0.183 & 0.203 \\
\hline
\end{tabular}

Table 1 The selected cases are reported with relevant quantities.

In the following, for sake of simplicity, $t / T=0$ corresponds to the first zero crossing-up of the undisturbed incident wave at the cylinder axis (without cylinder) in stationary Fourier conditions. The analysis is here conducted on an integer number of incident wave periods, free from transient effects induced typically by the wave front and by possible reflections from the absorbing beach. When needed, averages over corresponding time steps $i+t / T$ are given, where $0 \leq i<4$. The cases presented here are given in Table 1; please note that in the present study the test number corresponds to the same test number given in Contento and Codiglia [16]. 


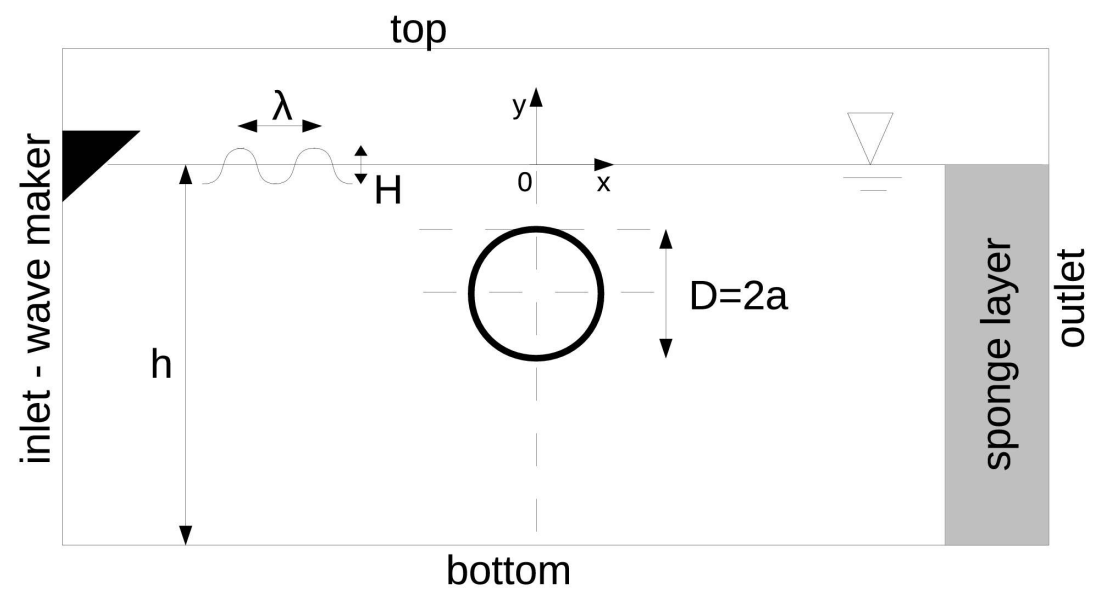

Fig. 1 A schematic representation of the physical problem, with relevant symbols.

The simulations conducted here and the experiments described in [16] have been planned in such a way that the diffraction parameter $k a$, the Reynolds number Re and the Keulegan Carpenter number $K C$ (nominal values related to the unperturbed incident flow) are kept constant while varying the depth of the cylinder $y$. The non-dimensional depth of the cylinder axis has been set to $\mathrm{y} / \mathrm{a}=-2,-3,-4$ respectively. $k a$ and $K C$ have been varied in the ranges $0.125 \leq k a \leq 0.52$ and $0.1 \leq K C \leq 2.50$ respectively. The maximum Reynolds number $R e$ achieved is $3.5 \times 10^{4}$, still largely in nominal laminar regime. The cases reported in Table 1 of the current paper have been selected from the larger experimental dataset of corresponding Table 1 in [16] in order to cover the entire range of $K C$ available in the experiments and with a specific interest for the largest values of $K C$ where the experiments have shown a complex fluid-body interaction.

\section{Mathematical model and numerical method}

Without heat exchanges, the governing equations in differential form for an incompressible Newtonian fluid are the following:

$$
\begin{aligned}
& \frac{\partial\left(\rho u_{i}\right)}{\partial t}+\frac{\partial\left(\rho u_{j} u_{i}\right)}{\partial x_{j}}=\frac{-\partial p}{\partial x_{i}}+\frac{\partial}{\partial x_{j}}\left(\mu\left(\frac{\partial u_{i}}{\partial x_{j}}+\frac{\partial u_{j}}{\partial u_{i}}\right)\right)+F_{i}-\rho \alpha v_{d}(x) u_{i} \\
& \frac{\partial u_{i}}{\partial x_{i}}=0
\end{aligned}
$$

where $\rho$ is fluid density, $u_{i}$ is a velocity component, $p$ is the pressure, $\mu$ is the dynamic viscosity, $F_{i}$ are the body forces, $t$ and $x_{i}$ are the time and space independent variables. The last term in the RHS of Eq. (2) is an additional term that corresponds to the artificial damping that is applied in a selected region of the domain to dampen-out the incoming waves at the boundary at the opposite end of the wavemaker (sponge layer). The artificial viscosity $v_{d}(x)$ is function of the longitudinal position $x$, it is set to zero at a given location $x_{\text {start }}$ in the domain and goes to its final value at the outlet. $\alpha$ is the volume fraction, introduced later on in Eq. (5) and (6). The artificial viscosity function must grow smoothly at the beginning of the sponge layer so that reflections do not occur at that position. Here a cubic polynomial has been used: 


$$
v_{d}(x)=\left\{\begin{array}{cc}
0 & \text { if } x<x_{\text {start }} \\
a\left(x-x_{\text {start }}\right)^{3}+b\left(x-x_{\text {start }}\right)^{2}+c\left(x-x_{\text {start }}\right) & \text { if } x>x_{\text {start }}
\end{array}\right.
$$

and $a=-2\left(v_{d}, \max -M L\right) /(L)^{3}, b=-3 a L / 2$ and $c=M$.

The starting location of the sponge layer $x_{\text {start }}$ and $v_{d}, \max$ are user specified and allow a convenient manipulation of the parameters. $M$ is the slope of the cubic at the beginning of the numerical beach; $L$ is the length of the sponge layer. The intensity of the artificial viscosity depends upon the amount of the wave energy to be dissipated and therefore it is tuned. This instrument is non selective in terms of wave frequency but in practice the length $L$ of the sponge layer determines the lowest frequency damped, so in the case of multiple frequencies $L$ must be set conveniently longer than the longest wave length $\lambda$ in the input spectrum [20] ensuring a very good performance for the shorter waves too. In the present simulations the following setup has been used $v_{d}, \max =120, L=2 \lambda$ and $M=0.01$. For the cases simulated, we found a reflection coefficient not greater than $7 \%$, computed according to the linear approach of partially reflected waves [21].

Broadly speaking, for small Reynolds number, the Navier-Stokes equations above can be solved directly without any turbulence parameterization. When this additional modeling becomes necessary, a possible approach can be based on the reformulation of the equations in terms of Reynolds averages, adding new equations that redefine the eddy viscosity. The discussion on turbulence modeling is notoriously wide and is out of the purposes of this work. The problem faced in this work regards a two-phase flow (a coupled air-water system). The treatment of the free surface is here pursued with the well-known interface capturing VOF method of Hirt and Nichols [22].

Briefly, the idea is to use a scalar function $\alpha$ such that $0 \leq \alpha \leq 1$ to represent the phase of the fluid in each cell ( 0 or 1 , if only air or water are present in a control volume respectively), therefore for the viscosity and density the following equations hold:

$$
\begin{aligned}
& \mu=\mu_{\text {water }} \alpha+\mu_{\text {air }}(1-\alpha) \\
& \rho=\rho_{\text {water }} \alpha+\rho_{\text {air }}(1-\alpha)
\end{aligned}
$$

Following Rusche [23], we have used a modified transport equation with an additional convective term that serves to keep the interface sharp:

$$
\frac{\partial \alpha}{\partial t}+\frac{\partial\left(u_{i} \alpha\right)}{\partial x_{i}}+\frac{\partial\left(w_{i} \alpha\right)}{\partial x_{i}}=0
$$

where $w_{i}$ is an artificial velocity field that is directed normal to and towards the interface. The relative magnitude of the artificial velocity is determined with the following expression:

$$
w_{i}=K_{c} n_{i} \max \frac{n_{i} F}{S}
$$

where $K_{c}$ is an adjustable coefficient that determines the magnitude of the compression, $n_{i}$ is the interface unit normal vector, $F$ is the flux and $S$ is the surface area. These equations complete the mathematical formulation of the two phase flow model. In the following, the nominal free surface elevation (air-water interface) is referred to $\alpha=0.5$. 
Eq. $(2,3)$ are solved with a Finite Volume technique, using schemes up to a $2^{\text {nd }}$ order integration. The simulations are conducted within the OpenFOAM [24] framework. The pressure-velocity coupling is achieved using a Pressure Implicit with Splitting of Operator (PISO) algorithm. The Euler explicit scheme is adopted to march forward in time. The freesurface location is computed using the Multidimensional Universal Limited for Explicit Solution (MULES) method.

As far as the wave generation is concerned, OpenFOAM library has been enriched with specific packages for wave generation. Among the others, the contributes of Jacobsen et al. [25] and Higuera et al. [26] worth mentioning. However, in this work, the authors have adopted their own wavemaking procedure described in Lupieri and Contento [27]. Briefly, it is based on the Airy model, modified with a transfer function such that the target wave height is attained at the cylinder's axis station. An additional modification of the boundary condition has been implemented in order to optimize the mass transport at the wave making boundary during a complete cycle. After the process of calibration, the produced wave exhibits the desired height and harmonic composition at the desired locations.

In order to obtain accurate simulations, the discretization of the space through the multiblocks computing grids is designed to represent the height $H$ of the undisturbed wave with 80 points. The 2-D computing domain consists of $n \lambda$ before the cylinder and $m \lambda$ after the cylinder axes, with $n=4.5$ and $m=n+2$.

The first computing node at the wall of the cylinder has been placed at $y+=1$ estimated according to Schlichting formulation, which allows an accurate discretization of the boundary layer. The grading to the outer layer is imposed according to standard ITTC recommendations on CFD in Marine Hydrodynamics [28]. Grid sizes are around $1.8 \times 10^{6}$ cells and the computations have been conducted over a dedicated 48 cores system.

Output data over one complete wave period are given with an integer number of samples exactly. No modeling for turbulence has been adopted in the simulations here presented.

The grid resolution can be considered adequate in the outer layer and close to the cylinder, where detaching structures are originated. In case of wave breaking, occurring at $y / a=-2$ for $K C \geq 0.8$, a turbulent regime, with bubbles and air entrainment, is present locally at the airwater interface, approximatively from the cylinder axis. In this limited area a model for turbulence could be adopted since the smallest scales of the phenomenon are probably not sufficiently sampled with the resolution previously described. In this case, some items have to be considered: the most common models in the RANS approach are not actually suited for this specific application (2-D flow, air-water interface, turbulence regime localized only in restricted areas) and this could lead to a lack of accuracy for instance in the prediction of the breaking wave shape or position [27] basically for an overestimation of the eddy viscosity. In 3-D approaches of traveling and breaking waves, for instance within the Large Eddy Simulations framework, the computing cost result higher but still some lack in the prediction of breaking position can be experienced [29].

\section{Results and discussion}

In spite of its simplicity in terms of geometry and forcing terms, the problem deserves richness of physics. For this reason this section is consistently organized in different subsections, examining in depth selected details of the fluid-body interaction, namely the free surface and pressure behavior, integral wave loads and vortex dynamics, with systematic comparisons with experimental data from Contento and Codiglia [16]. 
These items have been selected here for their meaningfulness and for their capability to include/recover results from other authors, obtained both in laboratory or numerically with complex or simplified methods.

The simulations conducted in this study are two dimensional, thus they refer to an ideal set-up that is missing -among others- 3-D effects of waves traveling in a real lab (crossing and end effects) and the intrinsic 3-D nature of turbulence, mostly in the breaking area. Furthermore, the problem investigated is a time dependent phenomenon and the computing effort may become much more than relevant. With awareness of the limitations given by neglecting 3-D effects, the 2-D approach is considered here affordable and acceptable for the purposes of this specific study.

\subsection{Free surface elevation and dynamic pressure at the body surface}

The time/space behavior of the wave travelling past the submerged cylinder and of the dynamic pressure at the cylinder is introduced with a sequence snapshots in Fig. 2a-h. They represent quantities at time instants equally spaced in time by $T / 8$. The selected case is here Test \#7 of Tab. $1: K C=0.40$ with $y / a=-2$ (i), -3 (ii), -4 (iii).

As explained above, each value shown in the plots is an average value over an integer number of events at corresponding time instants in a steady spectral content, i.e. $i+t / T$, where $0 \leq i<$ 4. The pressure scale in the plots is 1 radius $=1 \mathrm{kPa}$. A positive pressure is plotted outside the cylinder surface, negative inside.

The agreement between numerical results and experiments is considered here appreciable, especially concerning the pressure field at the cylinder surface.

At a low $K C$ value, regardless of the cylinder depth, the wave-body interaction is dominated by inertia terms and strong non-linear effects induced by the decreasing depth of the cylinder are hardly distinguishable in the time or space domain.

Qualitatively speaking, the free surface profiles in Fig. 2a-h show higher order fluctuations that look depending on the cylinder submergence. Still the quantitative nature of these terms requires another type of analysis. The rest of the study concerning the free surface is thus conducted in the frequency domain.

Fig. 3 shows the non-dimensional amplitude of the first $\eta^{I}$ and second $\eta^{I I}$ Fourier components of the free surface elevation for $y / a=-2 \mathrm{Vs}$ the non-dimensional longitudinal position $x / a$ from the cylinder and for selected $K C$. A similar approach has been adopted by Grue [14] and by Contento and Codiglia [16]. In those cases the harmonics' amplitudes have been averaged at the lee side of the cylinder axis, i.e. $1 \leq x / a \leq 8.5$. In the present case they are explicitly shown Vs $x / a$.

As a general comment extended to the cases not shown here explicitly with plots, at first order there is no evidence of a strong interaction between the free surface and the cylinder in terms of diffraction effects. In the upper part of the range of $K C$ values investigated, mostly in the case $y / a=-2$, the low submergence of the cylinder induces breaking. This is witnessed by the departure of the non-dimensional $\eta^{I}$ from the unit.

At second order, diffraction effects appear for $K C<0.80$. This consists of a rapid growth of $\eta^{I I}$ with $x / a$ on the lee side of the cylinder. At higher $K C$ values, the curve tends to flatten out, i.e. $\eta^{I I}$ does not grow with $K C$. This saturation effect has been observed also by Grue [14], whose tests were performed for shallower cylinders, his deepest condition is our shallowest. In that case, the saturation phenomenon of the higher harmonic components was observed systematically in the range $K C<0.4$. 
In the upper part of the investigated $K C$ range, the numerical results and the experiments of Contento and Codiglia [16] exhibit a spreading in the results of $\eta^{I I}$ along the lee side of the cylinder. This result is difficult to read. The present simulations suggest that this effect is probably related to the detachment of vortices from the cylinder surface and to their interaction in complex patterns with the free surface, that was probably hard to capture within the limits of the laboratory instruments.

\subsection{Inertia loads and Morison's equation coefficients}

For low values of the diffraction parameter $k a$, wave loads are typically computed adopting the so-called Morison approach [18]. The well known Morison equation for a sectional element of a long cylindrical structure, reads as follows:

$$
F(t)=C_{m} \rho A \frac{\partial u}{\partial t}+\frac{1}{2} C_{d} \rho D u|u|
$$

where $F(t)$ is the inline force per unit length, $\rho$ is the fluid density, $A$ and $D$ are reference area and length respectively, $\frac{\partial u}{\partial t}$ and $u$ are the unperturbed acceleration and velocity of the incident inline flow. $C_{m}$ and $C_{d}$ are the inertia and drag coefficients respectively.

In the present application, with an incident (orbital) wavy flow in the vertical plane, Morison equation can be used in both horizontal and vertical directions.

It has been widely shown [17] that at very low $K C$ numbers $(K C<3), F(t)$ is thoroughly dominated by the inertia term and that for a deeply submerged circular cylinder with uniform (in space) time dependent flow field, the inertia coefficient loses the dependence on $K C$ and $R e$ and it becomes $C_{m} \approx 2$.

Once $F(t)$ is known from experimental measurements or numerical simulations and once the wave kinematics $\left(\frac{\partial u}{\partial t}, u\right)$ has been measured or computed without obstacle, $C_{m}$ and $C_{d}$ can be computed by a fitting procedure. In this context, the problem of an accurate wave kinematics is crucial, mostly in experimental tests. Indeed it requires additional measurements with LDV or PIV without obstacle and appropriate phasing of the time series, with or without obstacle. Alternatively, wave kinematics is derived according to a given wave model, using wave elevation as reference signal.

In the present numerical simulations, wave kinematics has been derived directly, with additional simulations, without obstacle, using the same driving signal at the wavemaker.

In the experiments of Contento and Codiglia [16], wave kinematics has been derived indirectly, using a linear wave model, using the measured unperturbed wave elevation at the cylinder axis as reference signal.

The results of $C_{m}$ from the present numerical simulations and from the experiments are reported in Fig. 4. Solid lines represent the second-order expansion of $C_{m}$ in $K C$; the coefficients of the expansion are derived by a least-square fitting through the experimental data of $C_{m}$. 

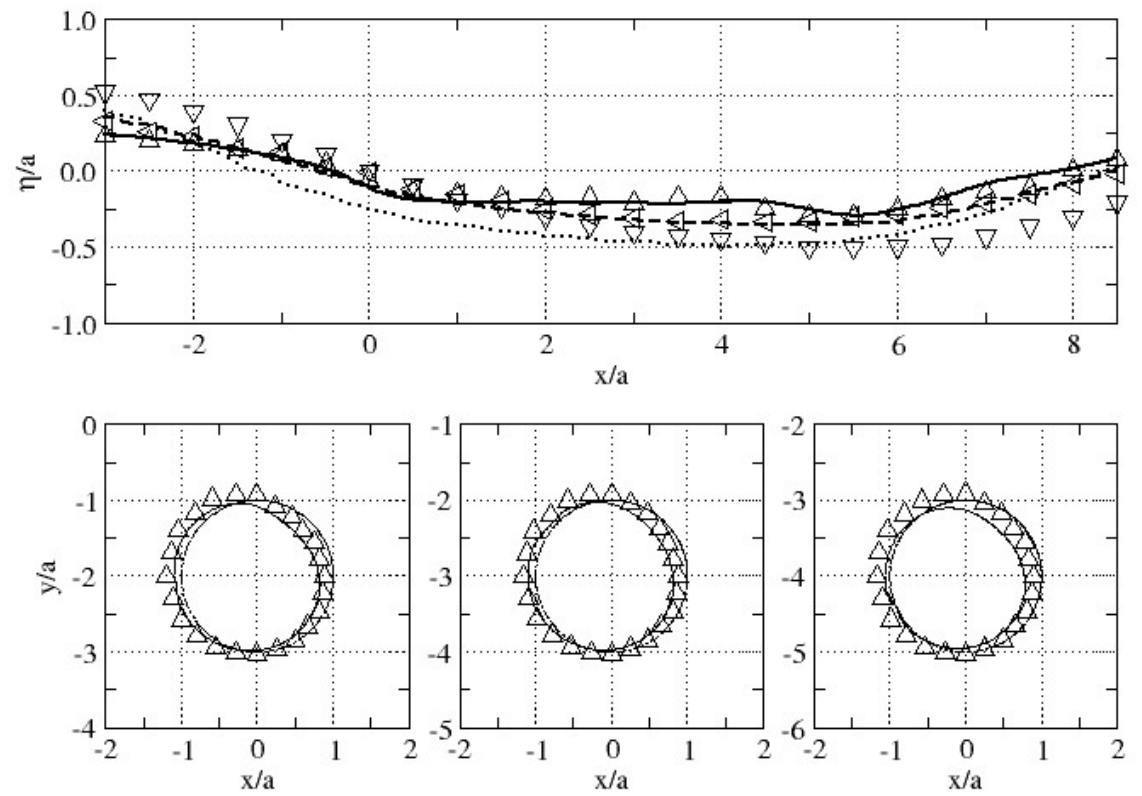

(a)
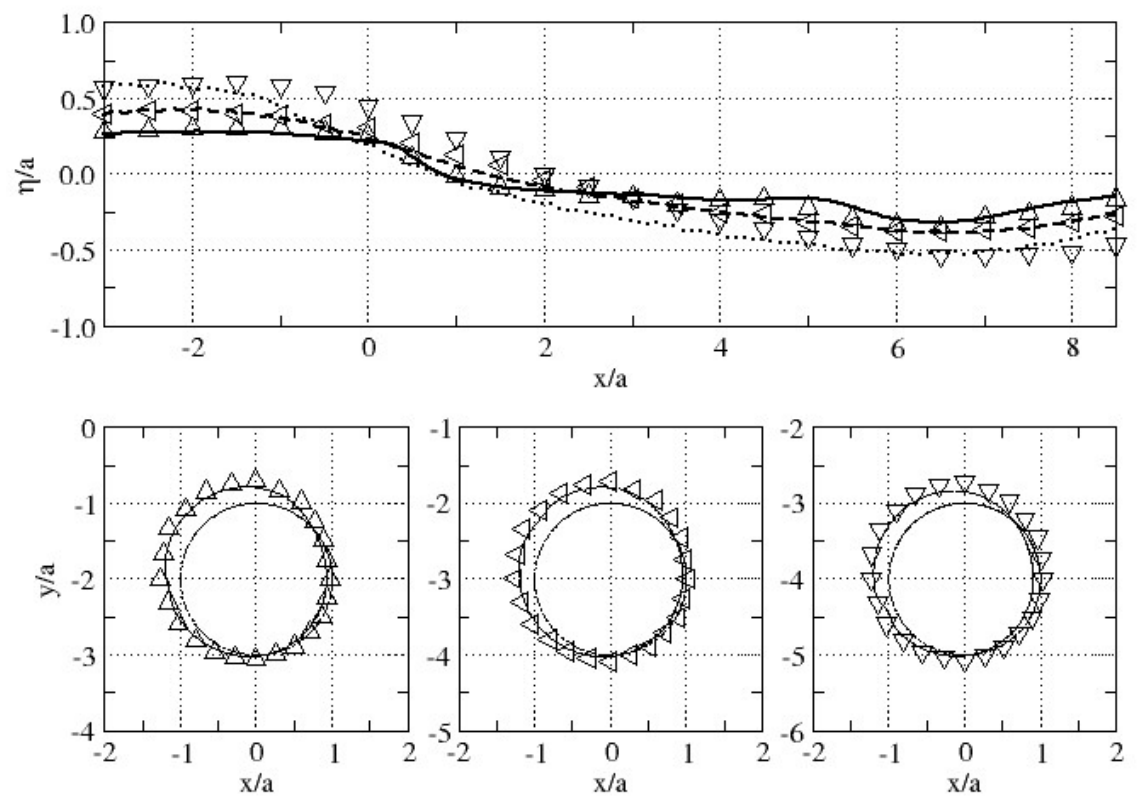

(b) 

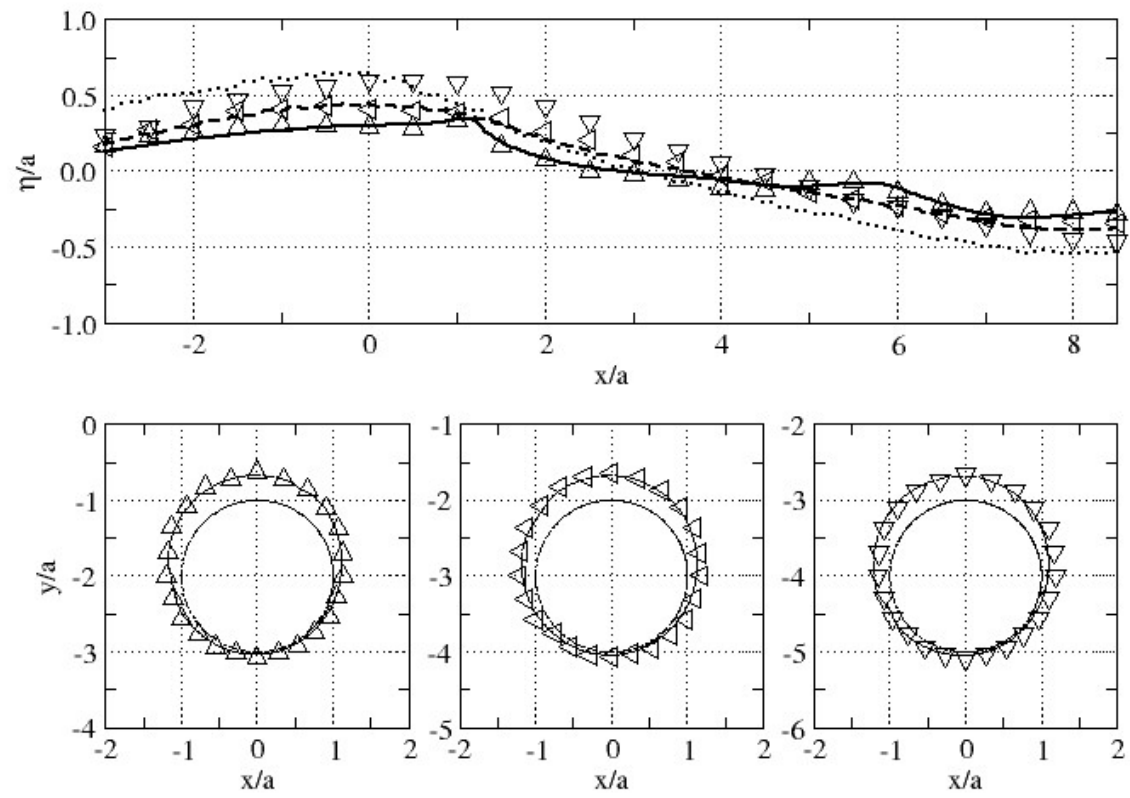

(c)
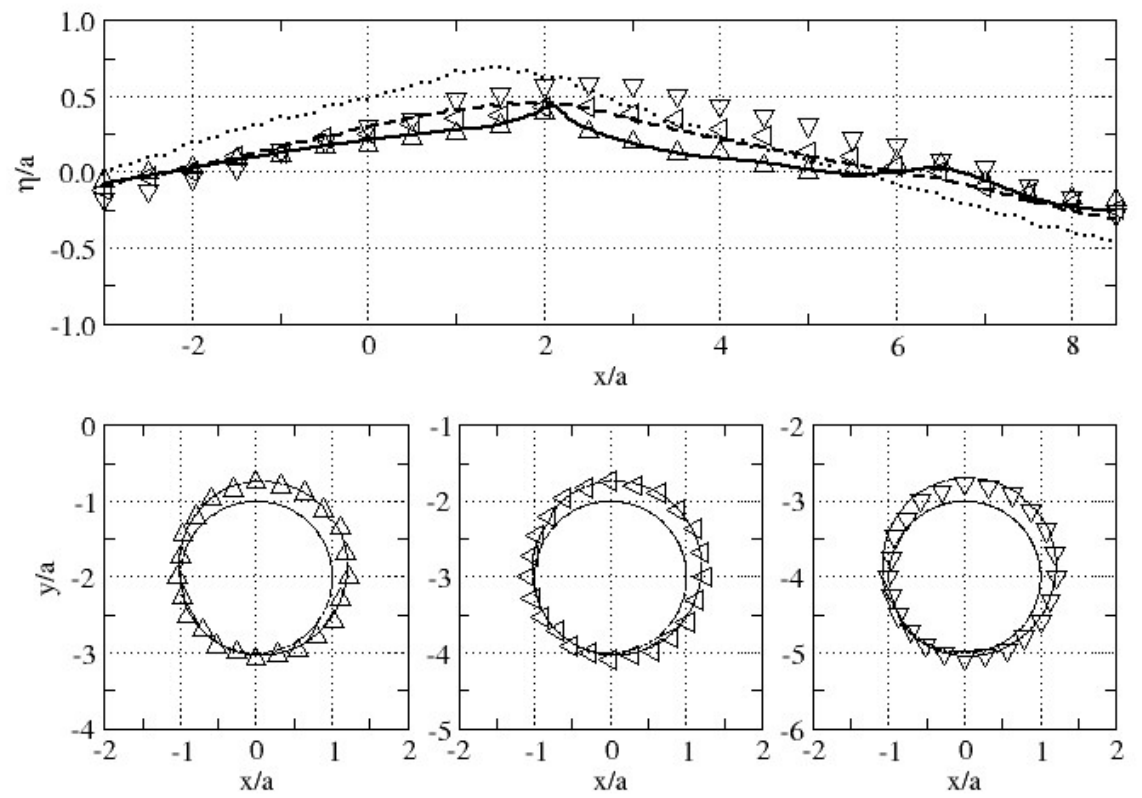

(d) 

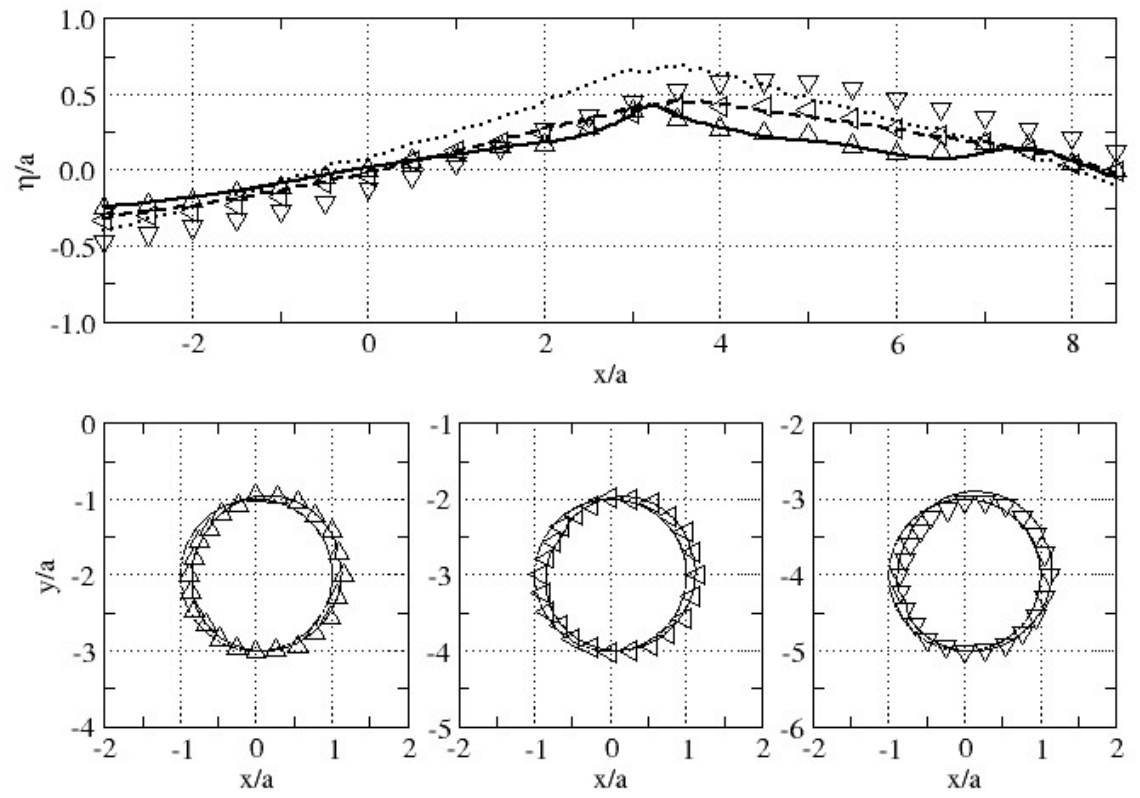

(e)
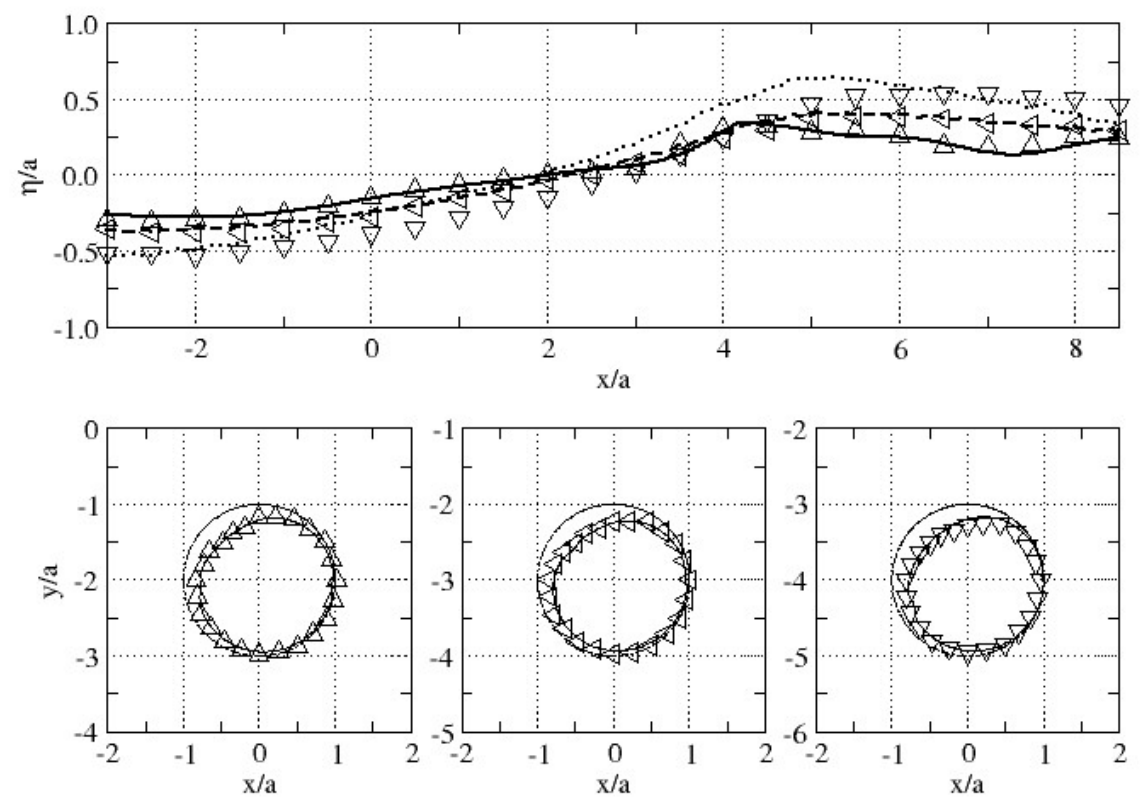

(f) 

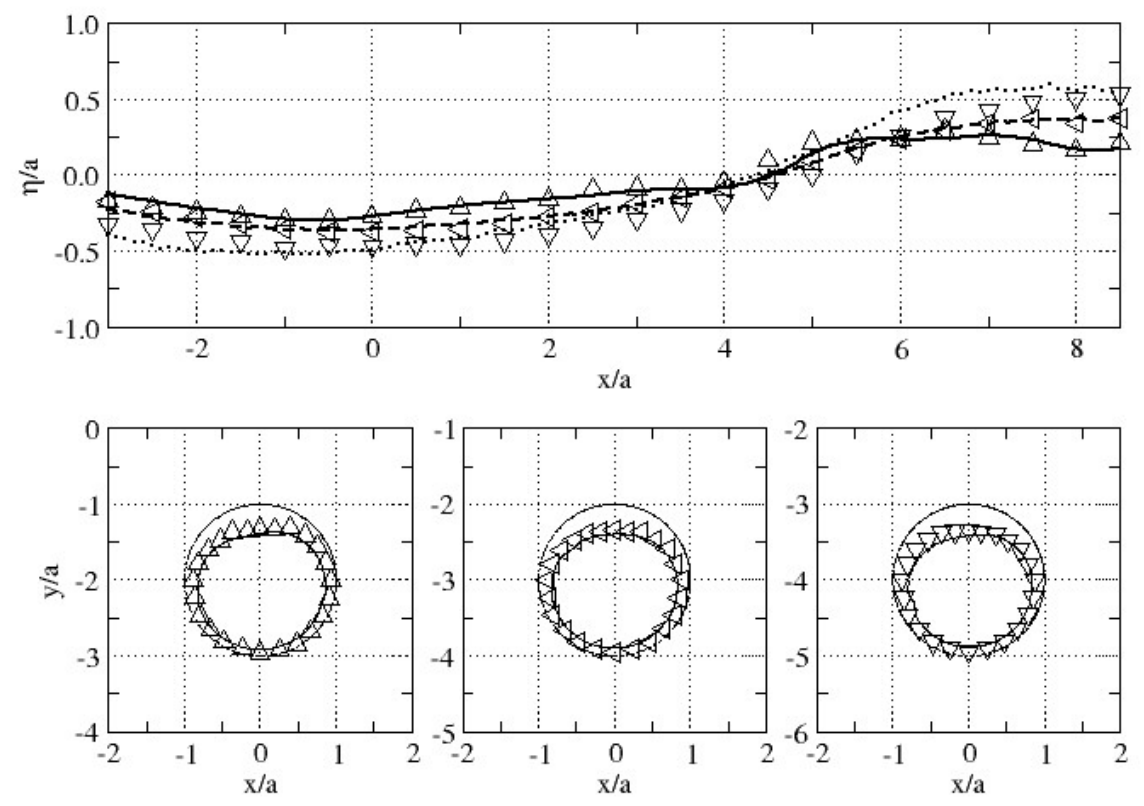

(g)
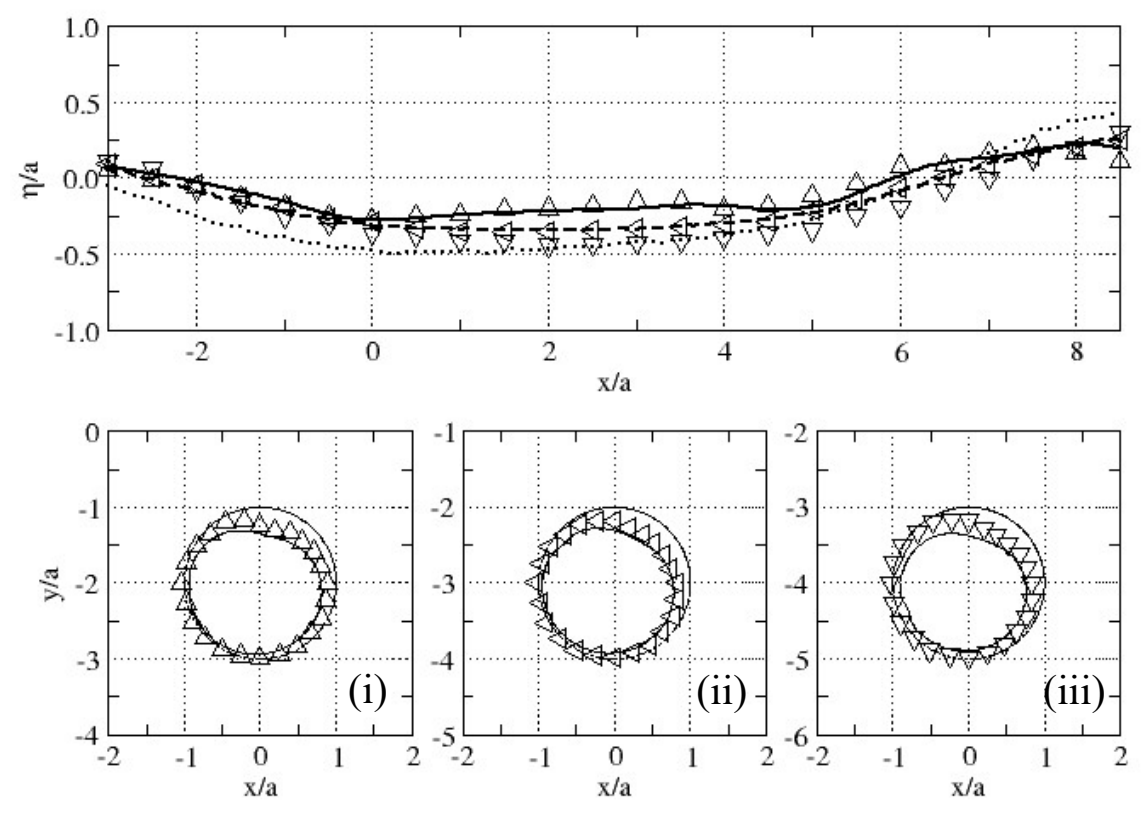

(h)

Fig. 2a-h Snapshots of the surface elevation (upper plot) and dynamic pressure at the cylinder surface (lower plots). Case $K C=0.40$. Lines $=$ numerical, symbols $=$ experimental.

$y / a=-2: \triangle$, solid line, (i); $y / a=-3: \triangleleft$, dashed line, (ii); $y / a=-4: \nabla$, dotted line, (iii). $t / T=0 / 8$, (b) $t / T=1 / 8$, (c) $t / T=2 / 8, \ldots$ (h) $t / T=7 / 8$. 1 radius $a=1 \mathrm{kPa}$. 

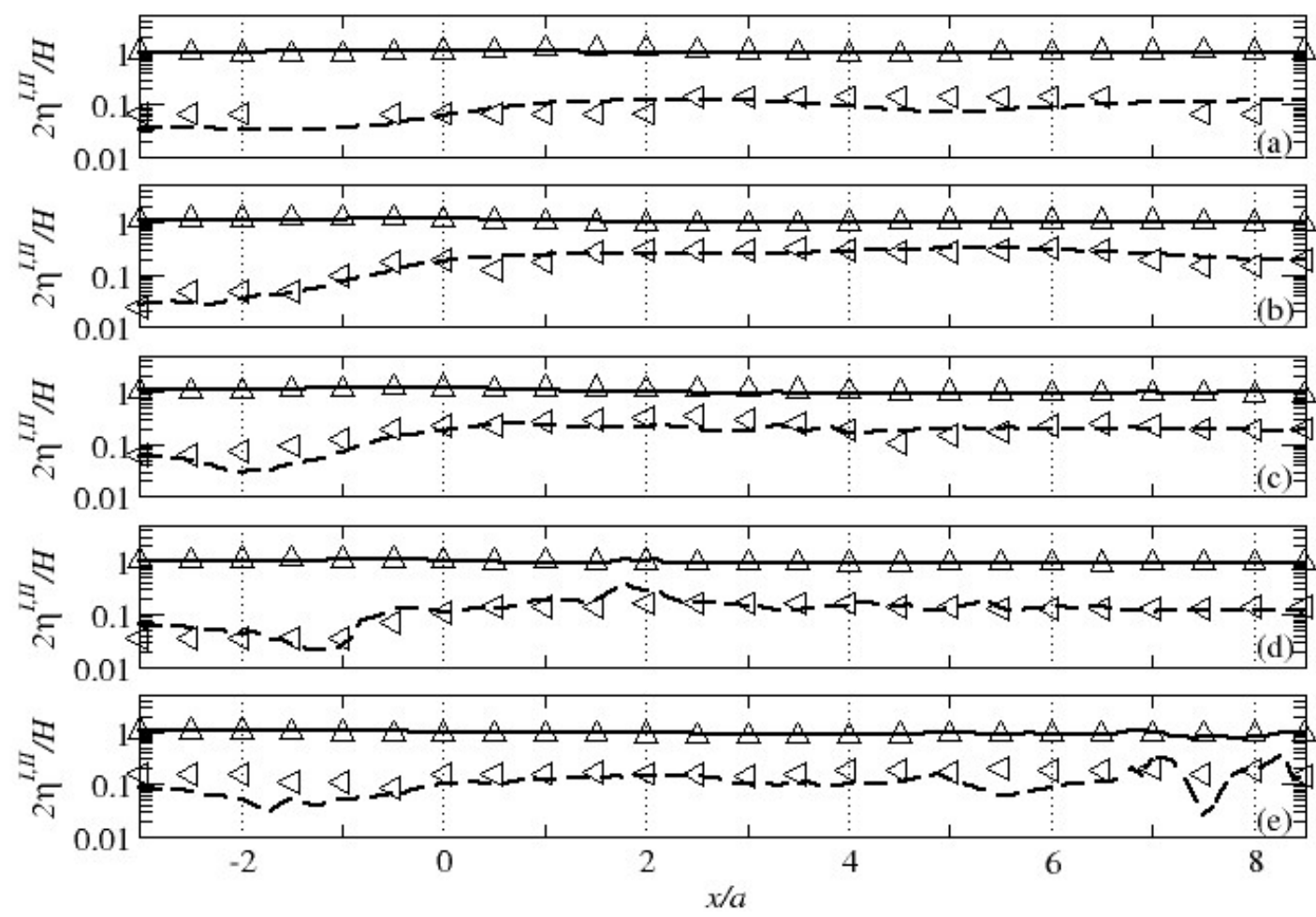

Fig. 3 Non-dimensional Fourier components $\eta^{I}$ and $\eta^{I I}$ of the free surface elevation at $y / a=-2$ Vs the nondimensional longitudinal position $x / a$.

$\eta I: \triangle=\exp$, solid line $=$ num; $\eta I I: \triangleleft=\exp$, dashed line $=$ num.

(a) $K C=0.10$, (b) $K C=0.40$, (c) $K C=0.80$, (d) $K C=1.54$, (e) $K C=2.50$ :

As consolidated in literature [9,10,11], the results obtained here confirm the rapid drop of $C_{m}$ from the limit value of 2 (approx.) for very small $K C$ to $C_{m} \approx 1$ or lower for $K C \approx 2$. This behavior of $C_{m}$ has been recognized to depend on viscous effects, i.e. steady rotating streaming that induces a Magnus effect between the steady rotating flow and the velocity component of the incident flow perpendicular to acceleration of the flow. Roughly speaking, the latter is inphase with the acceleration (max positive horizontal acceleration corresponds to a max positive vertical velocity) and, combined with the steady rotating flow, it produces a force that is in antiphase with the acceleration. As a result, the force in-lined with the acceleration is reduced considerably. 

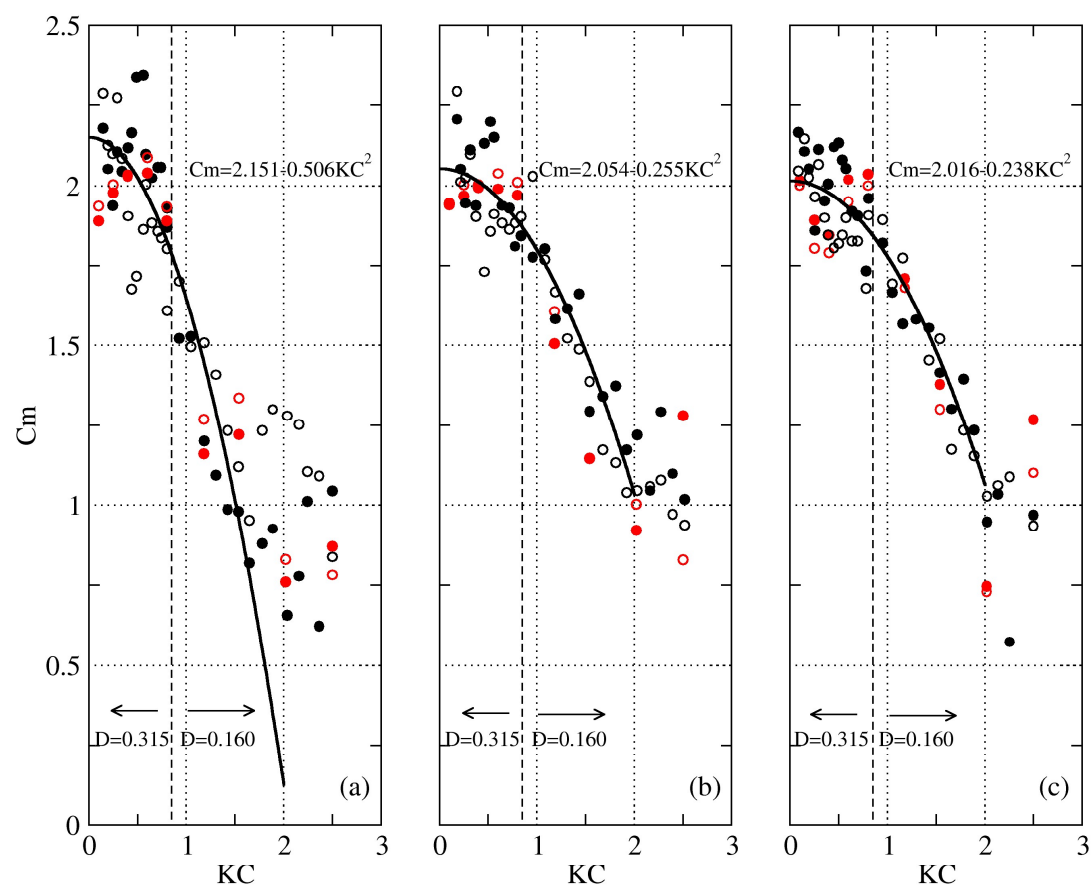

Fig. 4 Inertia coefficient $C_{m}$ Vs $K C$ for 3 cylinder submergences: $y / a=-2$ (a), $y / a=-3$ (b) and $y / a=-4$ (c). Solid and empty circles are horizontal and vertical components respectively. Black and red symbols are experimental and numerical values respectively.

\subsection{Effects of 2D turbulence on the eddies detached from the cylinders}

The experimental set-up used by Contento and Codiglia [16] could not cover all the aspects of the physics. Specifically, the eddies detachment, the eddies path and the relationship with the loads could not be determined by measurements.

In this section the focus is on the vorticity structures detached from the cylinder and three aspects relevant in turbulence dynamics: a) increase of vorticity intensity at the air-water proximity, b) vortex merging and c) persistence in the flow field.

The numerical simulations presented in this paper have been conducted in 2-D approximation, thus with a considerable reduction in the computing effort, with the aim to reproduce reasonably the main features of the flow as measured (namely the free surface and the wave loads). In this sense, the lack of the third dimension can be associated to a bare request of approximation in the free surface reproduction, thus excluding, at this stage, phenomena such as reflections from the lateral bulkheads (gentle but visible in the experimental sessions) and the 3D nature of spilling and plunging.

a) In the previous sections, the comparison of the free surface elevation and pressure at the cylinder surface has been made using averages over a selected number of periods for both measurements and numerical results. This technique may filter out the local fluctuations that eventually occur, thus allowing robust comparisons of the bulk of the flow, i.e. low order terms. On the other hand the presence of fluctuations in the flow fields is partially associated to the detachment of vorticity structures at the cylinder surface and partially to the large change of the wave shape before and at breaking. After detachment, these eddies can get closer to the free surface or remain in the cylinder proximity, subjected to the mean circulation surrounding the cylinder. See for instance Lupieri and Contento [27]. In the first case they produce an increment 
of the vorticity in the air-water interface. For $K C \geqslant 0.80$ it is observed that the vorticity present in the wave that is about to break is at least one order of magnitude smaller than that of the approaching detached vortices. As an example, Fig. 5 represents an incipient breaking wave and a detached vortex at the North of the cylinder for the case $K C \geqslant 1.54$ at $t / T=2.9$.

b) A second aspect here covered is vortex merging. Turbulence is an intrinsic 3-D phenomenon and a 2-D representation cannot include relevant aspects like tube vortex stretching or direct energy transfer from large to small scales. In spite of this, the interest for 2-D representations of turbulence remains wide and present, for instance, in the modelling of geophysical flows where it is convenient to consider that some flow features extend mainly in 2-D and can dominate part of the flow dynamics anyway. In this representation, eddies are observed to merge instead of splitting in smaller and smaller structures. The sequence of Fig. 6 taken from $K C=1.54$ and $y / a=-4$ witnesses the awaited merging. In this case 4 vortices are indicated with $\mathrm{A}, \mathrm{B}, \mathrm{C}$ and $\mathrm{D}$ and their evolution in time is followed with $d t \simeq T / 10$ up to the merge to obtain only two rotating structures.

c) In absence of merging with other eddies, the vorticity structures that depart from the cylinder proximity can exhibit persistency in time, while decreasing their vorticity intensity.

Likewise the wave period, the measure of eddies life (from detachment to dissolution) averaged over the whole ensemble of eddies detached reads as a relevant time scale of the phenomenon that it would have been interesting to evaluate but the most persistent structures show their presence for a time much longer than the conducted simulations.

Fig. 7 shows pairs of vorticity structures of both signs around the cylinder.

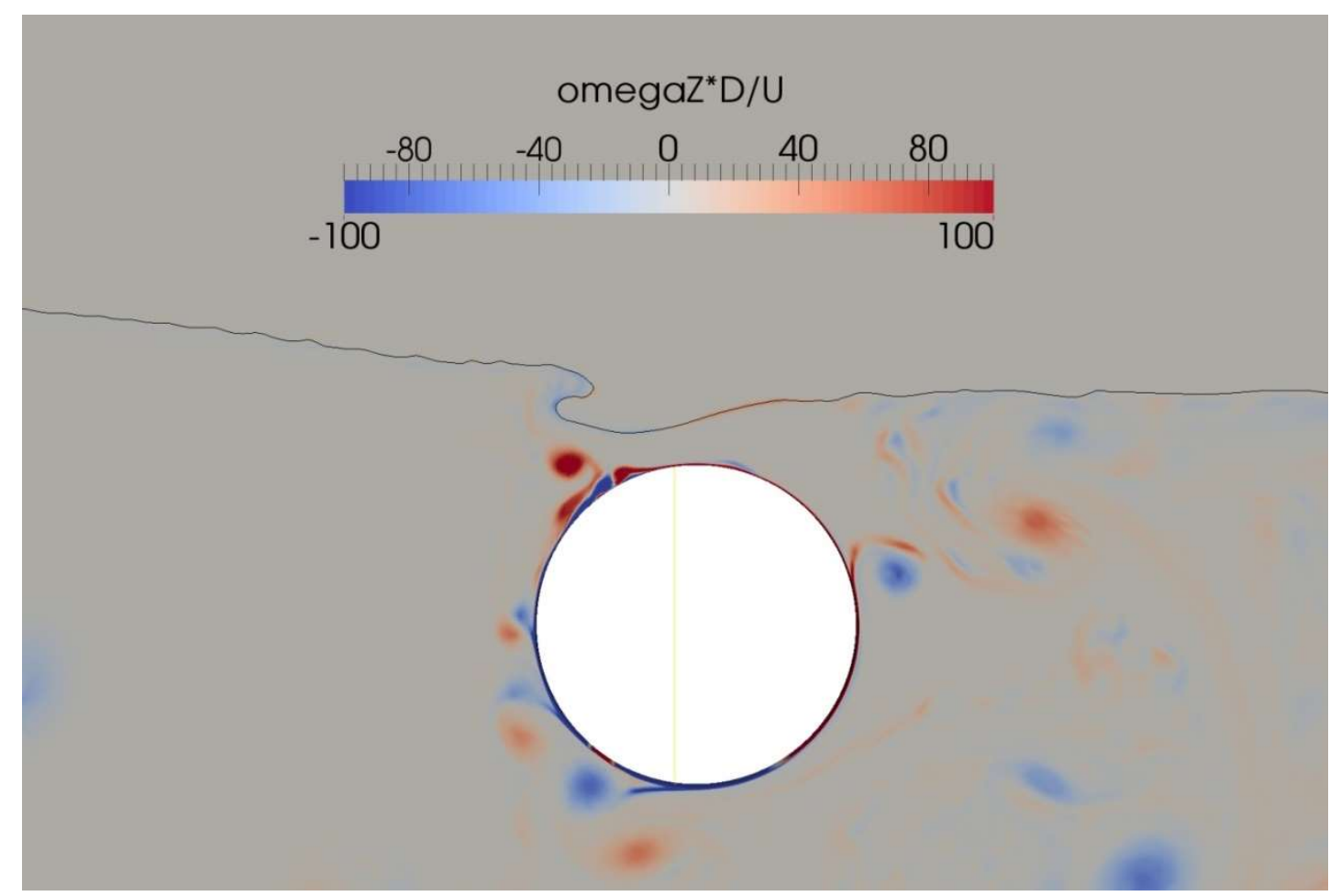

Fig. 5 A representation of the non-dimensional vorticity field in an area including the top of the cylinder and the incipient breaking free surface $(K C=1.54, y / a=-2, t / T=2.9)$. 


\section{Concluding remarks}

In this work the interaction of a weakly submerged horizontal circular cylinder with regular waves has been analyzed by means of numerical simulations supported by experimental data, in order to benefit from both methodologies in the attempt to explain the integral and local properties of the wave-body interaction.

The study has been conducted at low Keulegan Carpenter numbers $(K C \leq 2.5)$ and with a nominal Reynolds number in laminar regime $(\approx 100,000)$, varying the submergence of the cylinder. The study has been focused on some main aspects of the wave-body interaction, namely the pressure distribution on the cylinder surface, the free-surface elevation and finally some aspects on the vortex dynamics and free surface-vortex interaction.

Simulations and experiments show agreement in the quantities analyzed, such as free surface elevation and pressure at the cylinder. The evaluation of the inertia coefficient in the Morison equation remains critical but still the behavior of $C_{m} \mathrm{Vs} K C$ is reasonably well reproduced..

Last, room has been reserved to special 2-D effects nicely reproduced within the numerical approach. This has been a plus with respect to the experimental session, indicating that the two instruments of investigation can interpenetrate.

As a general and final comments, the numerical simulations here conducted have shown an good behavior with respect to the experimental data, furthermore the physics of this geometrically simple experiment remains rich and therefore, various additional insights

could be focused. By the numerics perspective, two further items could be the locally turbulent flow developed at the breaking region that probably requires a specific turbulence model and the determination of the contribute of each vortex on the forces acting on the cylinder, computed by means of the momentum of vorticity. 

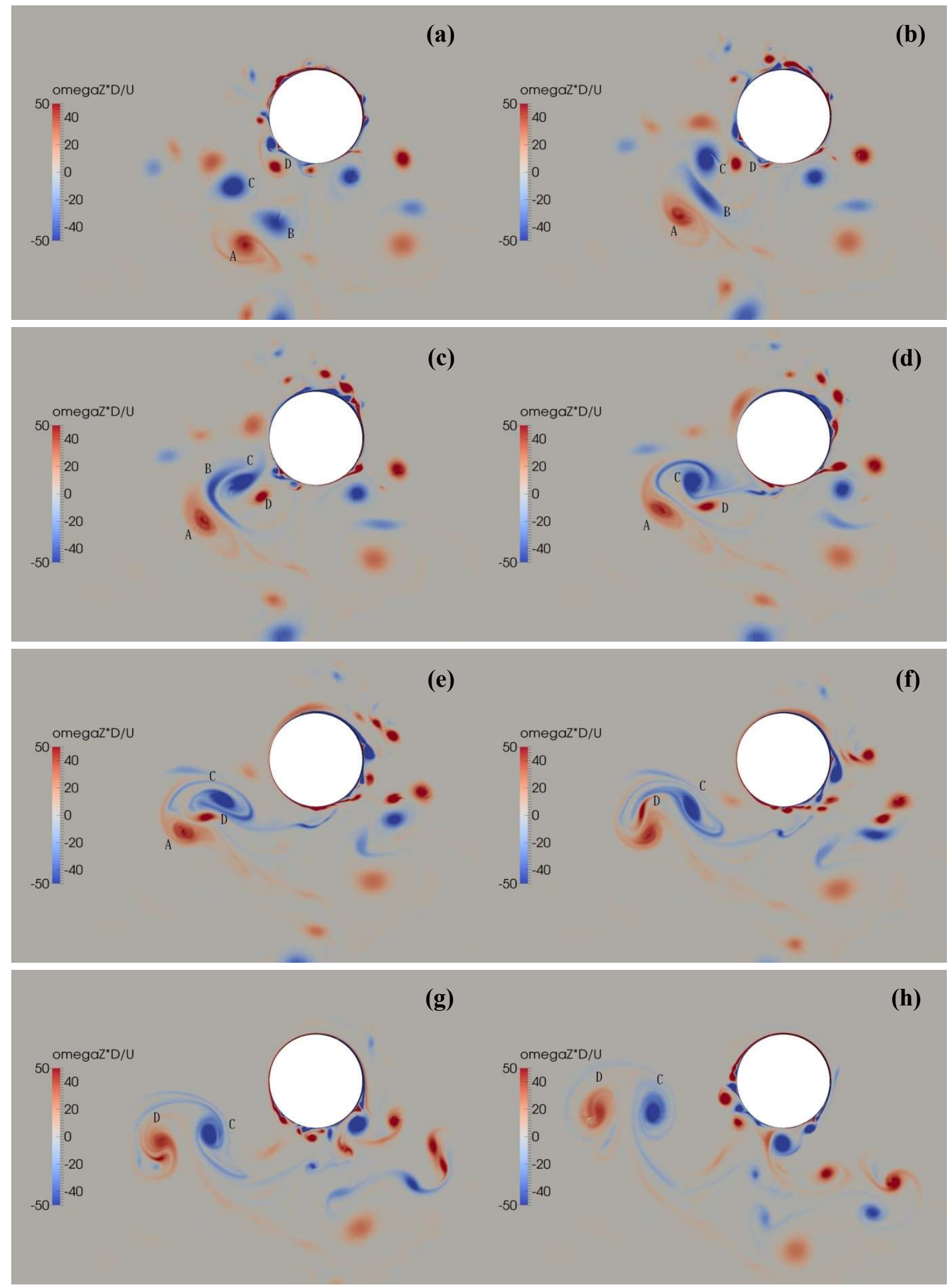

Fig. 6a-h A sequence of snapshots of the non-dimensional vorticity representing the evolution and merging of two pairs of vorticity structures at the cylinder proximity. ( $K C=1.54, y / a=-4$, $d t / T \approx 0.1)$. 4 vortices are indicated with $\mathrm{A}, \mathrm{B}, \mathrm{C}$ and $\mathrm{D}$ and their evolution in time is followed with $d t \simeq T / 10$. 


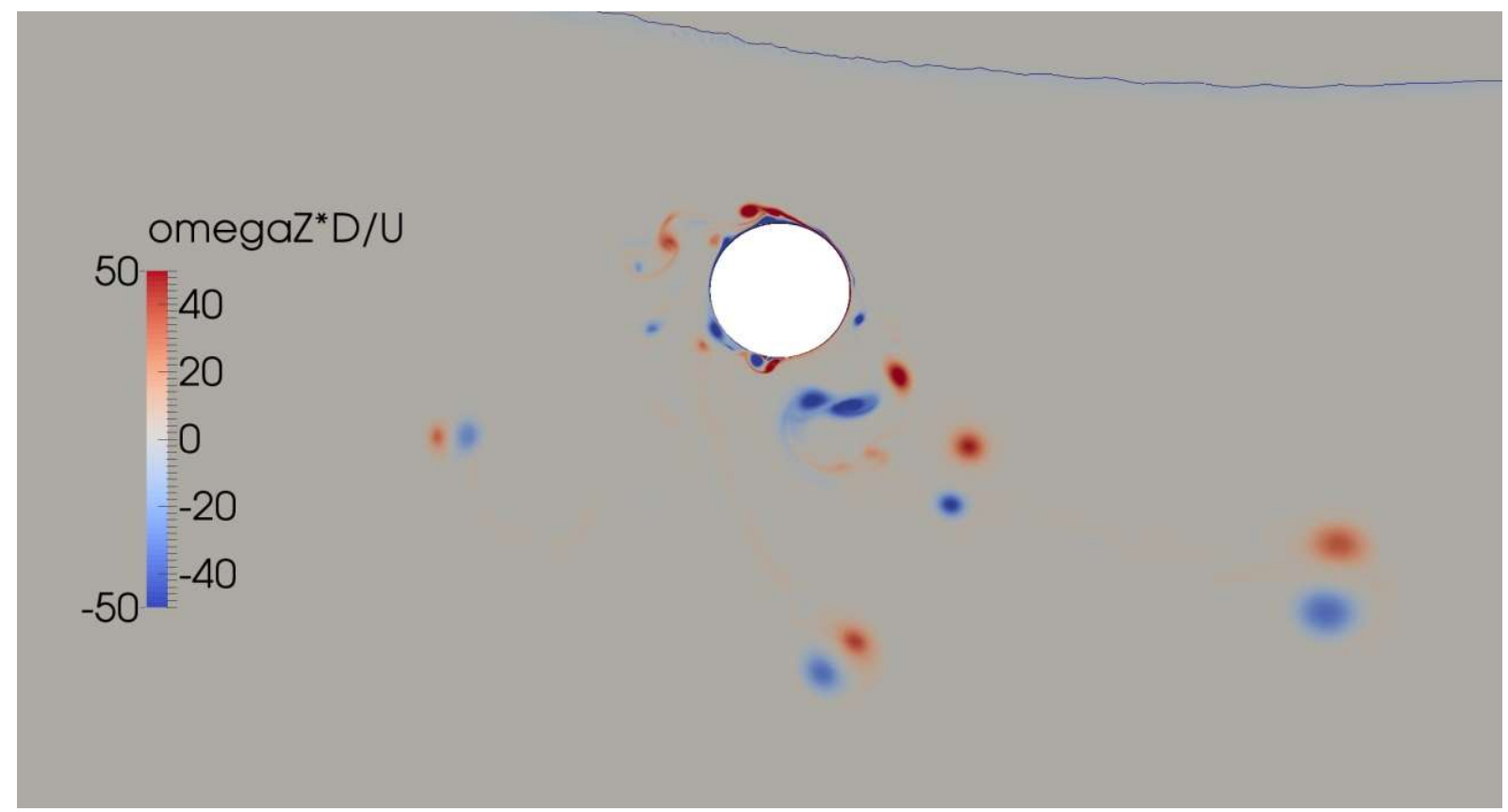

Fig. 7 A representation of pairs of the vorticity structures persistent in the flow field once released from the cylinder proximity.

\section{Acknowledgments}

The Programma Attuativo Regionale del Fondo per lo Sviluppo e la Coesione (PAR FSC 2007-2013 Linea d'Azione 3.1.2 is acknowledged for providing the financial support of the OpenViewSHIP Project.

\section{REFERENCES}

[1] Ogilvie, T.F., 1963. First and second order forces on a cylinder submerged under a free surface. Journal of Fluid Mechanics, Vol. 16, pp. 451-472. http://dx.doi.org/10.1017/S0022112063000896.

[2] Longuet-Higgins, M.S., 1970. Steady currents induced by oscillations around islands. Journal of Fluid Mechanics, Vol. 42, pp. 701-720. http://dx.doi.org/10.1017/S0022112070001568.

[3] Riley, N., 1971. Stirring of a viscous fluid. Z. angew. Math. Phys.,Vol. 22, pp. 645-653. http://dx.doi.org/10.1007/BF01587761.

[4] Riley, N., 1978. Circular oscillations of a cylinder in a viscous fluid. Z. angew. Math. Phys., Vol. 29, pp. 439-449. http://dx.doi.org/10.1007/BF01590765.

[5] Vada, T., 1987. A numerical solution of the second-order wave-diffraction problem for a submerged cylinder of arbitrary shape. Journal of Fluid Mechanics, Vol. 174, pp. 23-37. http://dx.doi.org/10.1017/S0022112087000028.

[6] Stansby, P.K. and Smith, P.A., 1991. Viscous forces on a circular cylinder in orbital flow at low Keulegan-Carpenter numbers. Journal of Fluid Mechanics, Vol. 229, pp. 159-171. http://dx.doi.org/10.1017/S0022112091002987.

[7] Wu, G.X. and Eatock Taylor, R., 1990. The second order diffraction force on a horizontal cylinder in finite water depth. Applied Ocean Research, Vol. 12, pp. 106-111. http://dx.doi.org/10.1016/S0141$1187(05) 80001-X$

[8] Stansby, P.K., 1993. Forces on a circular cylinder in elliptical orbital flows at low Keulegan-Carpenter numbers. Applied Ocean Research, Vol. 15, pp. 281-292. http://dx.doi.org/10.1016/01411187(93)90017-R.

[9] Otsuka, K. and Ikeda, Y., 1996. Estimation of inertia forces on a horizontal circular cylinder in regular and irregular waves at low Keulegan-Carpenter numbers. Applied Ocean Research, Vol. 18, pp. 145-156. http://dx.doi.org/10.1016/0141-1187(96)00023-5. 
A Numerical Study on the Viscous Effects of Waves Travelling

Past a Weakly Submerged Cylinder

Guido Lupieri, Giorgio Contento

[10] Chaplin, J.R., 1984a. Mass transport around a horizontal cylinder beneath waves. Journal of Fluid Mechanics, Vol. 140, pp. 175-187. http://dx.doi.org/10.1017/S0022112084000562.

[11] Chaplin, J.R., 1984b. Forces on a horizontal cylinder below waves. Journal of Fluid Mechanics, Vol. 147, pp. 449-464. http://dx.doi.org/10.1017/S0022112084002160.

[12] Chaplin, J.R., 1992. Orbital flow around a circular cylinder. Part 1. Steady streaming in non-uniform conditions. Journal of Fluid Mechanics, Vol. 237, pp. 395-411. http://dx.doi.org/10.1017/S002211209200346X.

[13] Chaplin, J.R., 1993. Orbital flow around a circular cylinder. Part 2. Attached flow at larger amplitudes. Journal of Fluid Mechanics, Vol. 246, pp. 397-418. http://dx.doi.org/10.1017/S0022112093000199.

[14] Grue, J., 1992. Nonlinear water waves at a submerged obstacle or bottom topography. Journal of Fluid Mechanics, Vol. 244, pp. 455-476. http://dx.doi.org/10.1017/S0022112092003148.

[15] Oshkai, P. and Rockwell, D., 1999. Free surface wave interaction with a horizontal cylinder. Journal of Fluids and Structures, Vol. 13, pp. 935-954. http://dx.doi.org/10.1006/jfls.1999.0237.

[16] Contento, G. and Codiglia, R., 2001. Non linear free surface induced pressure on a submerged horizontal circular cylinder at low Keulegan-Carpenters numbers. Applied Ocean Research, Vol. 23, pp. 175-185. http://dx.doi.org/10.1016/S0141-1187(01)00004-9.

[17] Keulegan, G. H.; Carpenter, L. H. (1958), "Forces on cylinders and plates in an oscillating fluid", Journal of Research of the National Bureau of Standards, Vol. 60 (5), pp. 423-440, http://dx.doi.org/10.6028/jres.060.043.

[18] Morison, J. R., O'Brien, M. P., Johnson, J. W., Schaaf, S. A., 1950. "The force exerted by surface waves on piles", Petroleum Transactions (American Institute of Mining Engineers) Vol. 189, pp. 149-154, http://dx.doi.org/10.2118/950149-G

[19] Chaplin, J.R. 2001. Non-Linear Wave Interactions with a Submerged Horizontal Cylinder. Proceedings of the Eleventh (2001) International Offshore and Polar Engineering Conference., Vol. III, pp. 272-279.

[20] Lupieri, G., Puzzer, T. and Contento, G., 2014. Numerical study of the wave-wave interaction by viscous flow simulation with OpenFoam. The 21 th Symposium Theory and Practice of Shipbuilding SORTA, Baska, Island of Krk, Croatia.

[21] Dean, R.G. and Dalrymple, R.N., 1984. Water Wave Mechanics for Engineers and Scientists. World Scientific Advanced Series on Ocean Engineering, Prentice Hall Inc., Englewood Cliffs NJ.

[22] Hirt C.W. and Nichols, B.D., 1981. Volume of fluid (VOF) method for the dynamics of free boundaries. J. Comput. Phys, Vol. 39, pp. 201-225. http://dx.doi.org/10.1016/0021-9991(81)90145-5.

[23] Rusche, H., 2002. Computational Fluid Dynamics of Dispersed Two - Phase Flows at High Phase Fractions. Imperial College of Science, Technology \& Medicine, London.

[24] OpenFOAM, 2012. OpenFoam user guide. OpenCFD Ltd.

[25] Jacobsen N.G., Fuhrman D.R., and Fredsøe J., 2012. A wave generation toolbox for the open-source CFD library: OpenFOAM. International Journal for Numerical Methods in Fluids, Vol. 70(9), pp. 10731088. http://dx.doi.org/10.1002/fld.2726.

[26] Higuera P., Lara L.J., and Losada I.J., 2013. Realistic wave generation and active wave absorption for Navier-Stokes models application to OpenFOAM. Coastal Engineering, Vol. 71, pp. 102-118. http://dx.doi.org/10.1016/j.coastaleng.2012.07.002.

[27] Lupieri, G. and Contento, G., 2015. Numerical simulations of 2-D steady and unsteady breaking waves. Ocean Engineering, Vol. 106, pp. 298-316. http://dx.doi.org/10.1016/j.oceaneng.2015.07.014.

[28] Specialist Committee on CFD in Marine Hydrodynamics, 2014. Proceedings - Volume II. 27 th International Towing Tank Conference (ITTC), Copenhagen.

[29] Christensen, E.D., 2006. Large Eddy Simulation of Spilling and Plunging Breakers. Int. Journal for Coastal, Harbor and Offshore Engineers, Vol. 53, pp. 463-485.

$\begin{array}{lll}\text { Submitted: } & 22.04 .2016 . & \begin{array}{l}\text { Guido LUPIERI, glupieri@units.it } \\ \text { Giorgio CONTENTO, contento@units.it }\end{array} \\ \text { Accepted: } & \text { 12.09.2016. } & \begin{array}{l}\text { Department of Engineering and Architecture, via Valerio, } 10-34127 \\ \text { University of Trieste, Italy }\end{array}\end{array}$

